\title{
Moral bioenhancement, freedom and reasoning
}

This issue includes a number of papers on reproductive ethics, broadly construed. In a recent book, Anja Karnein proposed that embryos created in vitro should be offered up for adoption before being discarded or used in research; ${ }^{1}$ here Timothy Murphy offers a critical response (see page 392). Elsewhere, Tak Chan and Stark \& Delatycki debate the role of medical professionals in providing parentage determination. Chan argues that doctors are obliged to provide parentage tests when this is requested by parents, provided there is a low risk that the child will be abandoned (see page 383). Stark $\&$ Delatycki discuss some difficulties raised by the 'risk of abandonment' condition (see page 387). Finally, papers by Kate Greasley and David Lang discuss the controversy surrounding the criminal prosecution of abortion doctor Kermit Gosnell. Greasley criticises the way in which some commentators capitalised on horror at Gosnell's extreme practices to advance more general anti-abortion objectives (see page 419). Lang argues, against Greasley, that the dominant pro-life response to the case was neither selfcontradictory nor intellectually dishonest (see page 424).

The issue is dominated, however, by discussions of 'bioenhancement' - the use of biomedical technologies to augment human capacities. A common criticism of bioenhancements is that they are antisocial: though they may benefit the user, they will have a negative effect on societyat-large. Such concerns are perhaps well placed in relation to the most obvious contemporary examples of bioenhancement: doping in sport, aesthetic medicine, and the use of stimulants to enhance examination performance. However, two varieties of bioenhancement discussed in this issue might be thought to have more positive social effects.

David Shaw discusses the possible use of cognitive enhancing drugs to improve health outcomes in disadvantaged segments of society (see page 389). Such enhancements might be thought to have desirable social effects insofar as they promote population level health and help to diminish health inequalities.

Meanwhile, David DeGrazia and four commentators debate the science and ethics of moral bioenhancements: bioenhancements that augment our moral capacities. Being moral and acting morally often (on some views, always) advance the common good, so moral bioenhancements might seem less susceptible than the most prominent existing forms of bioenhancement to the charge that they are antisocial. Nevertheless, a number of authors, including John Harris, Robert Sparrow and Nicholas Agar, have argued against moral bioenhancement, or certain varieties of it. In his feature article, DeGrazia comes to the defence of moral bioenhancement (see page 361, Editor's choice). In the remainder of this concise argument, I focus on DeGrazia's article and the commentaries on it.

\section{DEGRAZIA'S ARGUMENT}

DeGrazia sets the scene for his discussion by offering some examples of moral bioenhancement, highlighting some of its potential benefits, and laying out a perspicuous taxonomy. He then turns to an epistemic problem that has frequently been raised in debate in this area: given immense moral disagreement, how are we to determine what transformations qualify as moral bioenhancements? DeGrazia's proposal is that we classify as moral bioenhancements transformations that correct what all reasonable moral views would deem to be moral defects, which he takes to include, for instance, intrinsic delight in cheating others, deficits in empathy, and prejudice against outsiders. He suggests that we already implicitly make such an appeal to consensus among reasonable moral views in deciding what forms of moral education to permit in schools, and what forms of putatively immoral conduct to punish through criminal justice.

DeGrazia next turns to consider the moral desirability of moral bioenhancement. Much of his argument here is a response to John Harris' claim that moral bioenhancements of the kind discussed to date would undermine the enhanced individual's freedom. DeGrazia offers a wide ranging response in which he claims that none of the leading views on the nature of freedom support Harris' contention: moral bioenhancement need not threaten freedom where that is understood as requiring 'doing what one wants', 'being able to act otherwise' or being an 'unmoved mover'. He also offers his own account of free action and argues that, on that account too, our freedom could be unscathed by moral bioenhancement. Further, DeGrazia argues that even if moral bioenhancement did diminish freedom, it could still be morally desirable, all things considered, since the undesirable effect on freedom might be outweighed by other, desirable effects.

\section{RESPONSES}

Four commentators reply to DeGrazia's feature article.

Nicholas Agar focusses on a conceptual question: what is moral bioenhancement? (see page 369). DeGrazia understands it to be any improvement in moral capacities (of an individual or a population). But Agar suggests that we could understand it more narrowly, to include only transformations that improve moral capacities beyond a normal level. He then argues that attempts at moral bioenhancement, understood in this more limited way, are problematic in a way that attempts to bring someone up to normal moral competence are not. He claims that attempts to enhance beyond normal moral competence are likely to cause moral disenhancement, in part because they are likely to lead to imbalances in the capacities required for moral conduct.

Molly Crockett brings a neuroscientific perspective to the discussion (see page 370). She seeks to temper DeGrazia's optimism about the prospects for moral bioenhancement. Proponents of moral bioenhancement, DeGrazia included, often advert to neuroscientific studies finding that pharmacological agents have significant moral effects. But Crockett notes that most such studies have measured only one narrow psychological variable that might be thought relevant to morality, and have measured it only under laboratory conditions. They are arguably thus very far from establishing that any of the examples of moral bioenhancement offered by DeGrazia are or will soon be possible. Crockett also argues that most substances capable of modulating moral capacities are likely to have a wide range of side effects because the neurotransmitter systems thought to underpin moral capacities also perform many other roles. 
David Wasserman picks up on one of DeGrazia's positive arguments in favour of the desirability of moral bioenhancement (see page 374). DeGrazia suggested that improving moral behaviour would make the world a better place and improve the lives of its inhabitants. But Wasserman, invoking a nonconsequentialist moral view, argues that in certain contexts immoral behaviour has good social effects: we often need imorally ruthless politicians and cold professionals in order to realise positive social outcomes. He does not claim that we should never attempt to morally improve ourselves or our children, but he does suggest that the state, which plausibly has an obligation to make the world a better place, should allow beneficial moral defects to arise in a small percentage of the population.

Finally, John Harris offers a clarification and defence of his views regarding moral bioenhancement and freedom (see page 371). On the basis of this clarification, it seems that Harris and DeGrazia agree that moral bioenhancement exerts a causal influence on choices. They also agree that such influences could in principle diminish freedom. However, they disagree on how commonly they would do so. DeGrazia is of the view that moral bioenhancements will diminish freedom only in exceptional cases, while Harris thinks more-or-less the opposite. Although he does not hold that all forms of moral bioenhancement would diminish freedom, he maintains that "methods so far advanced by neuroscientists and some philosophers of neuroscience do seem inevitably to have this effect". Those methods involve the administration of pharmaceutical agents which influence, for example, impulsive agression, aversion to causing harm, willingness to co-operate with others, and xenophobia.
Harris' main basis for this concern appears to be that moral bioenhancement "bypasses reasoning" and "acts directly on attitudes". On his view, an action is free (and indeed qualifies as a truly moral action) only if arrived at through reasoning. But in arguing that moral bioenhancement would not normally threaten freedom, DeGrazia did not explicitly consider such an account of freedom.

\section{POSSIBLE REPLIES}

Suppose that Harris is right to claim that free actions must issue from reasoning. Might DeGrazia nevertheless be able to sustain his position? There seem to be at least two possible lines of response open to him here.

First, he might appeal to ways in which moral bioenhancements could operate precisely by improving moral reasoning processes, for example, by attenuating motives that tend to infere with sound moral reasoning. Harris appears to assume that moral bioenhancements would typically diminish or even eliminate the role of reasoning in producing actions. But perhaps they would often operate by enhancing reasoning. On Harris' account of freedom, such interventions would plausibly increase freedom, not decrease it.

Second, DeGrazia might press Harris to offer a fuller specification of how, precisely, reasoning must contribute to action in order for that action to be free. There may be scope for DeGrazia to develop a dilemma for Harris here.

On the one hand, Harris might take a hard line, maintaining that, to qualify as free, an action must be wholly the product of reasoning. But then DeGrazia could invoke his discussion of the ways in which ordinary 'unenhanced' moral agents appear to be pushed around by causal forces that do not involve reasoning. This discussion suggests that none of our actions are wholly the product of reasoning. Thus, if Harris takes this hard line, he may be forced to accept the unpalatable conclusion that none of our actions are free.

Alternatively, Harris could take a softer line which allows that actions can be free even if partly produced by processes other than reasoning. He might, for example, require only that reasoning must have played some role in producing the action, that the mental processes which led up to the action were initiated by reasoning, or that the final decision to undertake an action was made through reasoning. However, then it will no longer be clear why moral bioenhancement would typically, or even frequently, diminish freedom. Moral enhancements can certainly leave the enhanced agent with the capacity to make final decisions about how to act on the basis of reasoning: they might operate on mental states further back in the aetiology of the action. In such cases, the requirement that reasoning played some role in producing the action would also be met. And we might even plausibly say that actions brought about through moral bioenhancement were initiated by reasoning in cases where the decision to undergo the enhancement was itself made on the basis of reasoning.

Perhaps Harris can escape the second horn of this dilemma, but he will need to do so, I think, by offering a fuller account of the role of reasoning in producing free action that is both plausible, as an account of freedom, and such that moral bioenhancements would typically prevent reasoning from fulfilling that role.

\section{REFERENCE}

1 Karnein AJ. A theory of unborn life: from abortion to genetic manipulation. New York: Oxford University Press, 2012. 